\title{
A naked-eye triple system with a nonaccreting black hole in the inner binary ${ }^{\star}, \star \star$
}

\author{
Th. Rivinius ${ }^{1}$, D. Baade ${ }^{2}$, P. Hadrava ${ }^{3}$, M. Heida ${ }^{2}$, and R. Klement ${ }^{4}$ \\ 1 European Organisation for Astronomical Research in the Southern Hemisphere (ESO), Casilla 19001, Santiago 19, Chile \\ e-mail: triviniu@eso.org \\ 2 European Organisation for Astronomical Research in the Southern Hemisphere (ESO), Karl-Schwarzschild-Str. 2, \\ 85748 Garching b. München, Germany \\ 3 Astronomical Institute, Academy of Sciences of the Czech Republic, Boční II 1401, 14131 Praha 4, Czech Republic \\ 4 The CHARA Array of Georgia State University, Mount Wilson Observatory, Mount Wilson, CA 91023, USA
}

Received 25 March 2020 / Accepted 22 April 2020

\begin{abstract}
Several dozen optical echelle spectra demonstrate that HR 6819 is a hierarchical triple. A classical Be star is in a wide orbit with an unconstrained period around an inner $40 \mathrm{~d}$ binary consisting of a B3 III star and an unseen companion in a circular orbit. The radial-velocity semi-amplitude of $61.3 \mathrm{~km} \mathrm{~s}^{-1}$ of the inner star and its minimum (probable) mass of $5.0 M_{\odot}\left(6.3 \pm 0.7 M_{\odot}\right)$ imply a mass of the unseen object of $\geq 4.2 M_{\odot}\left(\geq 5.0 \pm 0.4 M_{\odot}\right)$, that is, a black hole $(\mathrm{BH})$. The spectroscopic time series is stunningly similar to observations of LB-1. A similar triple-star architecture of LB-1 would reduce the mass of the BH in LB-1 from $\sim 70 M_{\odot}$ to a level more typical of Galactic stellar remnant BHs. The BH in HR 6819 probably is the closest known BH to the Sun, and together with LB-1, suggests a population of quiet BHs. Its embedment in a hierarchical triple structure may be of interest for models of merging double $\mathrm{BHs}$ or $\mathrm{BH}+$ neutron star binaries. Other triple stars with an outer Be star but without $\mathrm{BH}$ are identified; through stripping, such systems may become a source of single Be stars.
\end{abstract}

Key words. stars: black holes - binaries: spectroscopic - stars: individual: HR 6819 - stars: individual: ALS 8775 (LB-1)

\section{Introduction}

The largest discrepancy between Galactic population-synthesis models and observations probably concerns the number of black holes (BHs). Models predict $10^{8}$ to $10^{9}$ stellar mass BHs (Agol \& Kamionkowski 2002; Olejak et al. 2020), but merely a few $10^{2} \mathrm{X}$-ray binaries are known, most of which only harbor neutron stars (NS). This comparison is severely biased because it only includes actively accreting BHs. Nonaccreting $\mathrm{BHs}$ are much more difficult to find (for a recent report of a detection, see Thompson et al. 2019). Compared to plain singlelined spectroscopic binaries (SB1), the presence of a second luminous star as a distant third body in a hierarchical triple can provide further constraints on the parameters of nonaccreting BHs (Hayashi et al. 2020). Massive hierarchical triple systems recently attracted interest because the outer object might accelerate the merger of inner double-BH or $\mathrm{BH}+\mathrm{NS}$ binaries through Lidov-Kozai oscillations, as detected through gravitational waves (Abbott et al. 2019, and references therein).

The system ALS 8775 (LB-1 in the following) was recently reported to host an apparently nonaccreting $\mathrm{BH}$ with the unusually high mass of $\sim 70 M_{\odot}$ (Liu et al. 2019). This is not easily reconciled with binary evolution and Galactic population-synthesis

$\star$ The authors dedicate this Letter to the memory of Stan Štefl (1955-2014) in sadness and grateful appreciation of his never-tiring alertness that also triggered this work.

$\star \star$ Based partly on observations collected at the European Southern Observatory, Chile (Prop. Nos. 63.H-0080 and 073.D-0274). models (Eldridge et al. 2019). While the validity of the mass determination involving emission lines has been questioned (e.g., Abdul-Masih et al. 2019; El-Badry \& Quataert 2020), a $\mathrm{BH}$ of more regular mass can still be inferred from the binary mass function derived from the luminous component. However, none of the rebuttals so far have offered a convincing explanation for the lack of motion of the broad $\mathrm{H} \alpha$ emission line, which in fact shows a profile that is very typical for Be stars.

HR 6819 (also known as HIP 89605, HD 167128, or QV Tel) appears as a bright $(V=5.3 \mathrm{mag})$ early-type Be star. Classical Be stars (Rivinius et al. 2013) are extremely rapid rotators, and their emission lines originate from a rotationally supported equatorial disk. Dachs et al. (1981) reported narrow absorption lines of Ca II and Si II as well as in He I $\lambda 4471 \AA$, which are uncommon in Be stars, and Slettebak (1982) noted a similarity of the absorption spectrum to that of a normal sharp-lined B3 giant. In spectra from 1999, Maintz (2003) found that these narrow lines actually are the signature of a second star with variable radial velocity (RV). Because of the small number of observations, she was only able to propose an orbital period of a few dozen days. In 2009, Hadrava, Stefl, and Rivinius disentangled the spectra of the two stars from a larger dataset, using the method of Hadrava (1995, 2004a), and discovered that HR 6819 contains a third, unseen object that may be a $\mathrm{BH}$. The results of this analysis will be presented in a forthcoming paper (in the following referenced as Hadrava et al., in prep.).

This Letter follows a more conventional and alternative approach. Section 2 derives the orbital parameters of the inner 
Table 1. Spectroscopic datasets.

\begin{tabular}{|c|c|c|c|c|c|c|c|c|}
\hline $\begin{array}{l}\text { Data } \\
\text { set }\end{array}$ & $\begin{array}{c}\text { Observing } \\
\text { season }\end{array}$ & $\begin{array}{c}\text { JD } \\
-2400000 \\
\end{array}$ & Telescope & Instrument & $\begin{array}{c}\text { Number of } \\
\text { spectra }\end{array}$ & $\begin{array}{c}\text { Resolving } \\
\text { power }\end{array}$ & $\begin{array}{c}\text { Spectral } \\
\text { range }[\AA]\end{array}$ & Ref. \\
\hline A & 1999 & $51373-51394$ & ESO $1.52 \mathrm{~m}$ & FEROS & 12 & 48000 & $3700-9000$ & 1,2 \\
\hline B & 2004 & $53138-53273$ & $\mathrm{ESO} / \mathrm{MPG} 2.2 \mathrm{~m}$ & FEROS & 51 & 48000 & $3700-9000$ & 2 \\
\hline
\end{tabular}

Notes. They are available from the LSW Heidelberg (Set A: https://www.lsw. uni-heidelberg.de/projects/instrumentation/Feros/ ferosDB/search.html) and the ESO Science Archive Facility (Set B: http://archive.eso.org/cms.html), respectively.

References. 1 - Maintz (2003), 2 - this work.

binary and estimates the space trajectory of HR 6819 as a whole relative to the Solar System. Section 3 argues that the unseen component is a BH, compares HR 6819 to other hierarchical triple systems with an outer Be star, identifies the similarity of the HR 6819 and LB-1 systems in all architectural details, and briefly sketches the history of HR 6819 relative to Sco OB2.

\section{Observations and their analysis}

\subsection{Time-resolved spectroscopy and photometry}

The findings by Maintz (2003) from dataset A in Table 1 motivated further observations of HR 6819 for several months in 2004 (dataset B in Table 1). The instrument used was the echelleformat Fibre Extended Range Optical Spectrograph (FEROS, Kaufer et al. 1999). The spectra were reduced with the standard FEROS pipeline ${ }^{1}$.

HR 6819 is photometrically variable with a full range of nearly $0.1 \mathrm{mag}$. Time series from three different space photometers are described and discussed in Appendix B. No regularly repeating variability was detected.

\subsection{Inner orbit}

Figure 1 and Appendix A.1 present the RVs of the sharp-lined star for which the FOTEL code of Hadrava (2004b) yielded the orbital parameters of the inner binary compiled in Table 2. Because dataset B spans several cycles, the orbital period is easily constrained to be about $40.3 \mathrm{~d}$ based on datasets A and B. FOTEL gives a period of $40.333 \pm 0.004 \mathrm{~d}$. A further and more detailed analysis that also includes historical data and evaluates the possibility of secular changes, will be provided by Hadrava et al. (in prep.) Because of the low eccentricity, the argument of the periastron, $\omega$, is poorly constrained $\left( \pm 20^{\circ}\right)$, and its errors propagate into those of the velocity amplitude, $\mathrm{K}_{1}$. Therefore $\omega$ was fixed, whereupon the errors on $\mathrm{K}_{1}$ dropped to the $1 \%$ level. In search for a possible orbital motion between 1999 and 2004 of the inner binary relative to the outer $\mathrm{Be}$ star, a third iteration also required common results from datasets $\mathrm{A}+\mathrm{B}$, but permitted the systemic velocity, $\gamma$, of the inner binary to be different for $\mathrm{A}$ and B. The difference $\gamma_{1999}-\gamma_{2004} \approx+2 \mathrm{~km} \mathrm{~s}^{-1}$ is not significant, in agreement with the positional invariance of the emission lines from the outer Be star (Figs. 2 and C.2; Hadrava et al., in prep.). Because the outer orbit is unknown, but may have a period of some decades and any orientation, an undetectably small orbital acceleration over a few years is very well possible, and the RV of the complete triple system is preliminarily adopted as the combined solution of sets $\mathrm{A}+\mathrm{B}, \gamma=+9.4 \pm 0.5 \mathrm{~km} \mathrm{~s}^{-1}$.

\footnotetext{
1 https://www.eso.org/sci/facilities/lasilla/ instruments/feros/tools/DRS.html
}

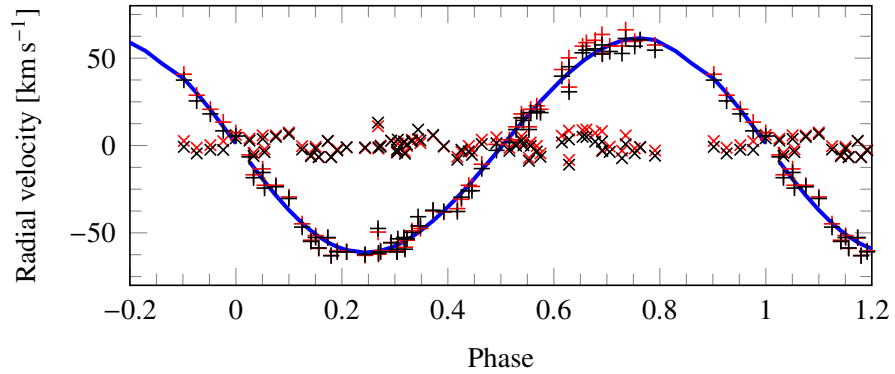

Fig. 1. RV curve (black pluses: He I $\lambda 4026$, red pluses: Mg II $\lambda 4481$ ) of the narrow-line star (see Appendix A and Fig. C.2) in the inner binary, the orbital fit (blue line; Table 2), and the residuals (crosses).

Table 2. Parameters of the inner binary in HR 6819 compared to those of the inner binary in LB-1 (Liu et al. 2019).

\begin{tabular}{|c|c|c|}
\hline Parameter & HR 6819 & LB-1 \\
\hline$[\mathrm{d}]$ & $40.333 \pm 0.004$ & 78.9 \\
\hline$\left[\mathrm{km} \mathrm{s}^{-1}\right]$ & $61.3 \pm 0.6$ & 52.8 \\
\hline L & $0.03 \pm 0.01$ & 0.03 \\
\hline [MJD] & $53177.44 \pm 0.07$ & N/A \\
\hline$\omega($ fixed $)$ & 89 & $\mathrm{~N} / \mathrm{A}$ \\
\hline$\left[\mathrm{km} \mathrm{s}^{-1}\right]$ & $9.4 \pm 0.5$ & 28.7 \\
\hline Mass function $f_{\mathrm{M}} \quad\left[M_{\odot}\right]$ & $0.96 \pm 0.03$ & 1.20 \\
\hline Minimum B star mass $\left[M_{\odot}\right]$ & 5.0 & 8.2 \\
\hline Minimum BH mass $\left[M_{\odot}\right]$ & $>4.2$ & $>6.3$ \\
\hline
\end{tabular}

Notes. The minimum BH mass for HR 6819 was derived with $f_{M}-2 \sigma=$ 0.90 instead of $f_{\mathrm{M}}$ itself to obtain a true lower limit.

\subsection{Spatial position and trajectory of the triple system}

Neither the Gaia (DR2 Gaia Collaboration 2018, $\pi=2.915$ mas) nor the HIPPARCos (van Leeuwen 2007, $\pi=4.29$ mas) parallax solutions for HR 6819 consider binarity; they are therefore highly uncertain. The Gaia measurements may suffer from the brightness of HR 6819 . However, at a distance of about $310 \mathrm{pc}$ (see below), the angular diameter of the circular orbit of the B3 III star of at least 0.44 au (for $\sin i=1$ ) is 1.4 mas or more. This matches the discrepancy as well as the astrometric excess noise of 0.731 mas in the Gaia solution. The observed flux is approximately compatible with both parallaxes. Depending on other assumptions made, a preference for a distance of about $310 \pm 60$ pc emerges from flux fitting (Appendix C).

The proper motion (in mas $\mathrm{yr}^{-1}:-3.667$ and -11.120 from Gaia and -4.10 and -13.30 from HIPPARCos in $\alpha$ and $\delta$, respectively) is much larger than the parallax and thus is less disturbed by any orbital pattern. At a distance of $310 \mathrm{pc}, 1$ mas $\mathrm{yr}^{-1}$ translates into $1.5 \mathrm{~km} \mathrm{~s}^{-1}$. When we average the Gaia and 

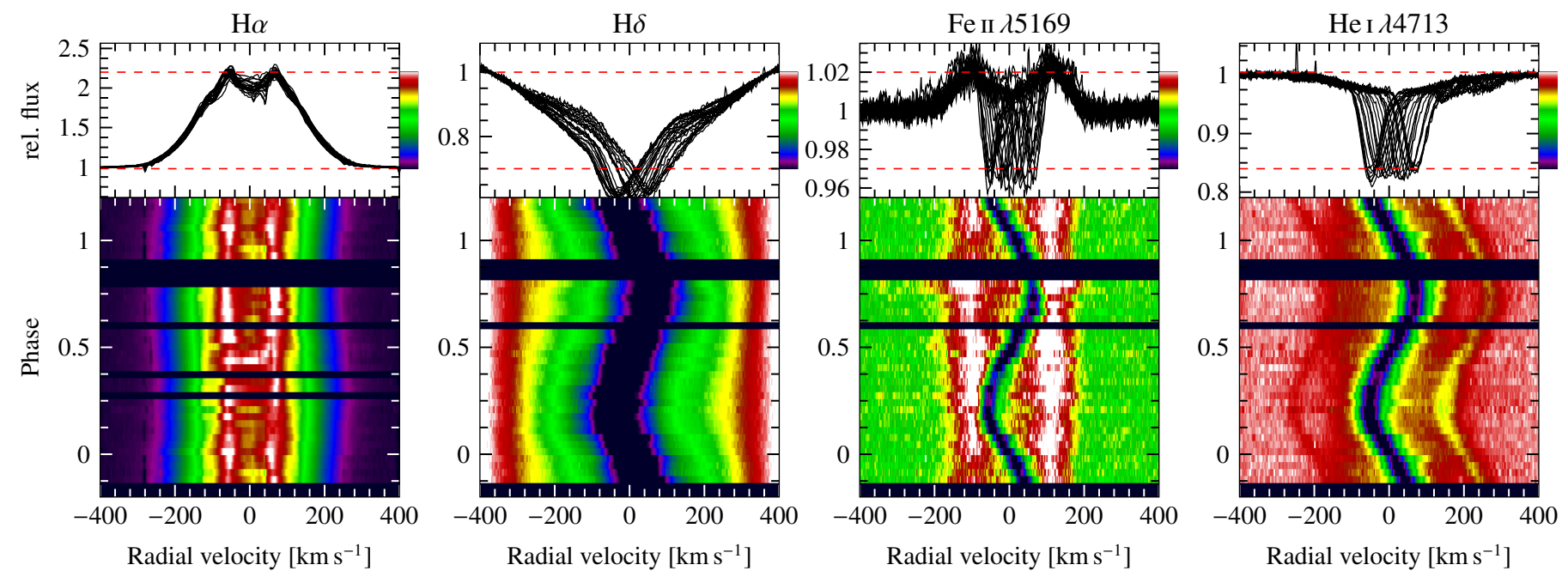

Fig. 2. Dynamical spectra of selected circumstellar and photospheric lines (as labeled) of the HR 6819 system, phased with the orbital period (see Table 2) of the inner binary. The individual line profiles are overplotted at the top of each panel; the dashed horizontal lines identify the range of the color-coding in the dynamical spectra. The $\mathrm{H} \alpha$ panel was constructed with data set B alone (see Table 1) to minimize the effect of the long-term disk variability of the Be star on the appearance of the emission. The basically invariant RV of the Be star is clearly visible in the peaks of its circumstellar emission lines (e.g., Fe II) as well as in the broad, weak absorption bases of predominantly photospheric lines such as He I. The cuts in the $\mathrm{H} \delta$ panels were chosen to highlight that at the $5 \%$ level (one tick mark in the upper panel) no feature moves in antiphase with respect to the line core. See Fig. C.2 for additional spectral lines.

HIPPARCos velocities as a simple estimate, the total lateral velocity is $12.8 \mathrm{~km} \mathrm{~s}^{-1}$, or $13.1 \mathrm{pc} \mathrm{Myr}^{-1}$, and the radial velocity of $9.4 \mathrm{~km} \mathrm{~s}^{-1}$ corresponds to $9.6 \mathrm{pc} \mathrm{Myr}^{-1}$. Without taking the $230 \mathrm{Myr}$ solar orbit in the Galaxy into account, that is, when we assume that the corotating frame is inertial, the closest approach of HR 6819 to the Sun is estimated for 11 Myr ago, at a distance of about $260 \mathrm{pc}$. A more precise trajectory and origin can only be derived when the outer orbit is known and the astrometric parameters have been computed taking the multiplicity into account.

\section{Discussion}

\subsection{Nature of the unseen inner component}

With the above orbital parameters, the mass function of the unseen component in the inner system becomes $0.96 \pm 0.03 M_{\odot}$ (see Appendix A for details). Evaluating a lower limit on the mass of the unseen component requires a lower limit on the mass of the B3 III star. Appendices C.1 and C.3 demonstrate that B3 III is sound as a spectral classification and agrees with the usage of that spectral type by Hohle et al. (2010). The database compiled by these authors contains 56 mass determinations for B3 III stars, none of which has a median value $<5.0 M_{\odot}$. This lower limit is also in agreement with evolutionary tracks (Appendix C.1). When we assume a lower limit of $5 M_{\odot}$ for the B3 III star and the $3 \sigma$ lower limit $\left(0.87 M_{\odot}\right)$ on the mass function, a hard lower limit on the mass of the unseen object is $4.2 M_{\odot}$.

Next, we need to verify whether a normal star with a mass at the lower limit can hide in the spectra of HR 6819. According to Hohle et al. (2010), the spectral type of stars with a typical mass of $4.2 M_{\odot}$ is about B7. For the lowest possible luminosity class of $\mathrm{V}$, and following Wegner (2006), the absolute visual magnitudes $M_{V}$ of a B3 III and a B7 V star differ by $1.7 \mathrm{mag}$, or a factor of almost 5 in flux at $550 \mathrm{~nm}$. Because the $\mathrm{B}$ and $\mathrm{Be}$ star both contribute about equally to the total $V$-band flux (Appendix C.3; Hadrava et al., in prep.), about $10 \%$ of the continuum would come from the inner companion if it were luminous. For this relative brightness, modeling with synthetic spectra from Shokry et al. (2018) shows that a B7 V star would appear with a spectral signature of $0.6 \%$ in $\mathrm{Mg}$ II $\lambda 4481$ and $6 \%$ in the Balmer lines even at near-critical rotation with $v \sin i>200 \mathrm{~km} \mathrm{~s}^{-1}$. That is, these features would not have gone unnoticed in the dynamical spectra that were constructed from more than 50 FEROs spectra with an average signal-to-noise ratio $S / N>280$ at $450 \mathrm{~nm}$, and the unseen component is not a normal star. Wang et al. (2018) searched UV spectra for an sdO companion. However, the $S / N$ of the calculated cross-correlation function was too low for a detection. Because the mass difference between the B star and the unseen object increases with the mass of the B star, the maximum mass of the unseen object also needs to be evaluated in principle because it might lead to a larger magnitude difference and hence lower detectability of a luminous companion. For HR 6819, however, this is not the case (see Appendix C.2).

HR 6819 is not positionally coincident with any known pul$\operatorname{sar}^{2}$. The lower limit of $4.2 M_{\odot}$ on the mass of the unseen object is substantially above the empirical mass limit of $\sim 2.6 M_{\odot}$ for NSs (Alsing et al. 2018, moreover, a companion mass close to that limit would imply an unbelievably low mass for the B star, see Appendix A) and also falls into the possible gap between observed masses of NSs and BHs (see Farr et al. 2011). Therefore, the unseen object must be a $\mathrm{BH}$.

Black holes are traditionally detected in X-rays that result from the accretion of matter from a companion star. By contrast, in the only recent X-ray observation of HR 6819, the ROSAT all-sky survey, the source is not detected $\left(L_{X} \leq 3.5 \times\right.$ $10^{30} \mathrm{erg} \mathrm{s}^{-1}$ in the $0.1-2.4 \mathrm{keV}$ band, assuming a distance of 460 pc; Berghoefer et al. 1996), and the near-circularity of the orbit does not suggest major variability in the form of undetected outbursts, as is confirmed in optical light (Appendix B). Accordingly, the inner binary is not interacting. At the temperature and

2 The ATNF catalog (Manchester et al. 2005) is maintained at https: //www.atnf.csiro.au/research/pulsar/psrcat/ 
luminosity of a B3 III star, radiative winds are intrinsically very weak (Krtička 2014) and are ineffective over a separation of $>0.22$ au (the lower limit from the circular inner B3 III-star orbit alone). Therefore, the lack of interaction is expected. This suggests that for all practical purposes, the $\mathrm{BH}$ is not accreting.

If, instead of the minimum mass of $5.0 M_{\odot}$ and the $3 \sigma$ lower limit on the mass function, we use the more typical mass of a B3 III star of $6.3 \pm 0.7 M_{\odot}$ (Appendix C.3) and the nominal value of the mass function, $0.96 \pm 0.03 M_{\odot}$, the $\mathrm{BH}$ mass increases from $4.2 M_{\odot}$ to $5.0 \pm 0.4 M_{\odot}$. For orbital inclinations $<90^{\circ}$ (the angle is unconstrained), both values would be higher.

\subsection{Similar systems}

As already noted by Liu et al. (2019), the shape of the line emission in LB-1 closely matches that of Be stars, although these authors only speak of "wine-bottle profiles" (which are typical for Be stars, see Hanuschik et al. 1996). The undetected RV amplitude of the emission line (El-Badry \& Quataert 2020) is naturally explained by a Be star at a large distance from the other luminous star, as in HR 6819. Table 2 compares the properties of the inner binaries in HR 6819 and LB-1. A forthcoming paper (Heida et al., in prep.) will disentangle the spectra of the two luminous stars in LB-1, which leads to a drastic reduction of the BH mass (see Abdul-Masih et al. 2019; El-Badry \& Quataert 2020).

In principle, both HR 6819 and LB-1 might be chance superpositions of a binary and a Be star, although this would stretch simple probability considerations. In both systems, the absolute and apparent spectroscopic luminosities of the visible components are similar (HR 6819: Appendix C, Hadrava et al., in prep.; LB-1: Heida et al., in prep.), and the similar distances support the assumption that both are physical triples.

The great similarity of HR 6819 and LB-1 invites the question whether this is enough to identify possible progenitor systems. The simplest assumption is that the hierarchy was not altered by the formation of the $\mathrm{BH}$, whereas the initial inner binary was tighter and had a total mass of well over $10 M_{\odot}$ (Sukhbold et al. 2016). One such candidate system may be the detached eclipsing $2.7 \mathrm{~d}$ binary CW Cep with masses of 13 and $12 M_{\odot}$ and a near-circular orbit (Johnston et al. 2019). Whether the two stars can avoid merging strongly depends on the mass loss. Johnston et al. (2019, their Fig. 2) reported broad H $\alpha$ emission that does not participate in the orbital motions, but has the typical profile of classical Be stars. This means that in CW Cep, an outer Be star may also be orbiting an inner binary. The $\mathrm{H} \alpha$ profile indicates an intermediate inclination of the Be star (see Hummel \& Vrancken 2000), while the orbital inclination of the inner binary is around zero because the system is eclipsing.

At first sight, it seems perplexing that all three systems host an outer Be star. Because HR 6819 and CW Cep were identified because their known line emission is similar to that in LB-1, this is probably a selection bias. However, 66 Oph is a fourth hierarchical triple with an outer Be star (but without a BH, Štefl et al. 2004). Such a channel for the formation of Be stars (for a description of other channels, see Langer et al. 2020a) may be due to angular momentum distribution during fragmentation in massive-star formation (Bodenheimer 1978; Kratter \& Matzner 2006). If these outer Be stars become unbound, they may have above-average space velocities, but probably not at the top of the range observed in a fair fraction of Be stars (Berger \& Gies 2001). Another conspicuous commonality is the vanishing eccentricity of the inner binaries, which is not typical of hierarchical triple systems (Tokovinin et al. 2019).

\subsection{Origin and history of HR6819}

The Solar System is located within the so-called Local Bubble, which measures about $100 \mathrm{pc}$ across and is characterized by a lower-than-average density, but high temperatures of its gas content (Slavin 2017). This structure is attributed to supernovae (SNe) that exploded several million years ago (as summarized by Frisch \& Dwarkadas 2017) when Sco OB2, the most nearby stellar OB association, passed in the solar vicinity (Fuchs et al. 2006). The analysis in Appendix D shows that HR 6819 is probably older than Sco OB2, and according to Sect. 2.3, its trajectory does not cross that of Sco OB2 (de Zeeuw et al. 1999), which therefore is not the parent population of HR 6819. Crude preliminary estimates of the distance of HR 6819 at the time of the $\mathrm{SN}$ explosion range from $280 \mathrm{pc}$ if the explosion occurred $15 \mathrm{Myr}$ ago to about $800 \mathrm{pc}$ for an explosion $\sim 65-70 \mathrm{Myr}$ ago (see Appendix D for a discussion of the age) and place this event well outside the Local Bubble. Depending on the initial mass, it is also possible that the $\mathrm{BH}$ formed without explosion by direct collapse (Sukhbold et al. 2016).

\section{Conclusions}

HR 6819 is a hierarchical triple star with a nonaccreting $\mathrm{BH}$ in the inner binary. The mass estimate does not depend on difficult RV measurements of emission lines as tracers of the orbital motion of the BH (see Casares et al. 2014; El-Badry \& Quataert 2020). Furthermore, the B3 III star is a fairly normal star so that the combination of the mass derived from spectral type and spectral energy distribution with the mass function leads to a solid lower mass limit on the $\mathrm{BH}$ of $4.2 M_{\odot}$ (for $\sin i=1$ ). The CW Cep system may be a progenitor of systems with an architecture similar as in HR 6819 and LB-1 if the $2.7 \mathrm{~d}$ inner binary can avoid merger through mass loss. Through stripping of the outer Be star, systems such as HR 6819, LB-1, CW Cep, and 66 Oph may become a source of single Be stars.

The detection was facilitated by the incompatibility of the spectral sequences with a simple binary. This can be a criterion to find other nonaccreting BHs, and the case of LB-1 illustrates the scope for such searches. If about $20 \%$ of all early-type stars are triples (see Moe \& Di Stefano 2017) and $0.01 \%$ of them have a system architecture similar to that of HR 6819 and LB-1, the discrepancy between expected and observed numbers of BHs in the Galaxy would shrink by several orders of magnitude but would still be very large. (In the magnitude-limited Bright Star Cata$\log$, Hoffleit \& Jaschek 1991, HR 6819 corresponds to $0.01 \%$ of all early- and late-type stars together.)

The existence of an entire population of quiet $\mathrm{BHs}$ is also suggested by the relative proximity of HR 6819. The lowest distance derived to date to any Galactic BH apparently is $<400 \mathrm{pc}$, which is the distance to the accreting V616 Mon (AO 0620-00; Foellmi 2009). The Gaia parallax of $0.64 \pm 0.16$ mas is closer to other distance estimates of $\sim 1 \mathrm{kpc}$ (Foellmi 2009), but would still make V616 Mon the nearest previously known BH to the Sun. At $310 \pm 60 \mathrm{pc}$, the distance to HR 6819 is far smaller than $1 \mathrm{kpc}$.

Triple systems have been invoked (Rodriguez \& Antonini 2018) as the progenitors of double BHs or BH + NS systems that merged owing to Lidov-Kozai oscillations triggered by a distant third object and were detected as gravitational-wave events (Abbott et al. 2019). It would therefore be interesting to compare dynamical models to HR 6819 or LB-1. However, the two luminous components of HR 6819 have masses of only $\sim 6 M_{\odot}$ and are very far apart, therefore the current $\mathrm{BH}$ will remain the 
only BH in HR 6819, and neither will an NS form. It appears noteworthy that the pure binary models of Langer et al. (2020b) predict a steep decrease in the fraction of $\mathrm{BH}$ companions toward the masses of the inner B stars in HR 6819 and LB-1. It may be useful to extend the models to triple systems.

HR 6819 does not share the proper motion of the young stellar associaton Sco OB2 and is probably also older than it. Any $\mathrm{SN}$ explosion leading to the $\mathrm{BH}$ formation most probably occurred outside the Local Bubble.

A subsequent paper (Hadrava et al., in prep.) will present the results of disentangling the spectra. The results are expected to achieve a comprehensive and largely independent quantitative confirmation of the properties of HR 6819 that we presented here.

Acknowledgements. This work was started when PH was a visitor at ESO, Santiago. $\mathrm{MH}$ is supported by an ESO fellowship. RKl acknowledges support by the National Science Foundation under Grant No. AST-1908026. This research has made use of NASA's Astrophysics Data System (ADS, Kurtz et al. 2000) and the SIMBAD database (Wenger et al. 2000) operated at CDS, Strasbourg, France. We are grateful for the referee's suggestion, which in particular led to an improved discussion in the appendices.

\section{References}

Abbott, B. P., Abbott, R., Abbott, T. D., et al. 2019, ApJ, 882, L24

Abdul-Masih, M., Banyard, G., Bodensteiner, J., et al. 2019, ArXiv e-prints [arXiv:1912.04092]

Agol, E., \& Kamionkowski, M. 2002, MNRAS, 334, 553

Aidelman, Y., Cidale, L. S., Zorec, J., \& Panei, J. A. 2018, A\&A, 610, A30

Alsing, J., Silva, H. O., \& Berti, E. 2018, MNRAS, 478, 1377

Baade, D., Rivinius, T., Pigulski, A., et al. 2016, A\&A, 588, A56

Baade, D., Rivinius, T., Pigulski, A., et al. 2018, in 3rd BRITE Science Conference, eds. G. A. Wade, D. Baade, J. A. Guzik, \& R. Smolec, 8, 69

Batten, A. H. 1973, in Binary and Multiple Systems of Stars, 1st edn. (Oxford: Pergamon Press), Int. Ser. Monogr. Nat. Philos., 51

Berger, D. H., \& Gies, D. R. 2001, ApJ, 555, 364

Berghoefer, T. W., Schmitt, J. H. M. M., \& Cassinelli, J. P. 1996, A\&AS, 118, 481

Bernhard, K., Otero, S., Hümmerich, S., et al. 2018, MNRAS, 479, 2909

Bodenheimer, P. 1978, ApJ, 224, 488

Bodensteiner, J., Sana, H., Mahy, L., et al. 2020, A\&A, 634, A51

Boggess, A., Carr, F. A., Evans, D. C., et al. 1978, Nature, 275, 372

Brott, I., de Mink, S. E., Cantiello, M., et al. 2011, A\&A, 530, A115

Cardelli, J. A., Clayton, G. C., \& Mathis, J. S. 1989, ApJ, 345, 245

Casares, J., Negueruela, I., Ribó, M., et al. 2014, Nature, 505, 378

Castelli, F., \& Kurucz, R. L. 2003, in Modelling of Stellar Atmospheres, eds. N. Piskunov, W. W. Weiss, \& D. F. Gray, IAU Symp., 210, A20

Dachs, J., Eichendorf, W., Schleicher, H., et al. 1981, A\&A, 43, 427

de Zeeuw, P. T., Hoogerwerf, R., de Bruijne, J. H. J., Brown, A. G. A., \& Blaauw, A. 1999, AJ, 117,354

Ekström, S., Georgy, C., Eggenberger, P., et al. 2012, A\&A, 537, A146

El-Badry, K., \& Quataert, E. 2020, MNRAS, 493, L22

Eldridge, J. J., Stanway, E. R., Breivik, K., et al. 2019, MNRAS, submitted [arXiv:1912.03599]

Farr, W. M., Sravan, N., Cantrell, A., et al. 2011, ApJ, 741, 103

Foellmi, C. 2009, New Astron., 14, 674

Frisch, P., \& Dwarkadas, V. V. 2017, in Effect of Supernovae on the Local Interstellar Material, eds. A. W. Alsabti, \& P. Murdin (Springer Internationa Press), 2253

Fuchs, B., Breitschwerdt, D., de Avillez, M. A., Dettbarn, C., \& Flynn, C. 2006 MNRAS, 373, 993
Gaia Collaboration 2018, VizieR Online Data Catalog: I/345

Hadrava, P. 1995, A\&AS, 114, 393

Hadrava, P. 2004a, Publ. Astron. Inst. Czechoslov. Acad. Sci., 92, 15

Hadrava, P. 2004b, Publ. Astron. Inst. ASCR, 92, 1

Hanuschik, R. W., Hummel, W., Sutorius, E., Dietle, O., \& Thimm, G. 1996, A\&AS, 116, 309

Haubois, X., Carciofi, A. C., Rivinius, T., Okazaki, A. T., \& Bjorkman, J. E. 2012, ApJ, 756, 156

Hayashi, T., Wang, S., \& Suto, Y. 2020, ApJ, 890, 112

Hick, P., Buffington, A., \& Jackson, B. V. 2007, in Solar Physics and Space Weather Instrumentation II, Proc. SPIE, 6689, 66890C

Hoffleit, D., \& Jaschek, C. 1991, The Bright Star Catalogue (Yale University Observatory)

Hohle, M. M., Neuhäuser, R., \& Schutz, B. F. 2010, Astron. Nachr., 331, 349

Howard, T. A., Bisi, M. M., Buffington, A., et al. 2013, Space Sci. Rev., 180, 1

Hummel, W. 1994, A\&A, 289, 458

Hummel, W., \& Vrancken, M. 2000, A\&A, 359, 1075

Irrgang, A., Desphande, A., Moehler, S., Mugrauer, M., \& Janousch, D. 2016 , A\&A, 591, L6

Johnston, C., Pavlovski, K., \& Tkachenko, A. 2019, A\&A, 628, A25

Kaufer, A., Stahl, O., Tubbesing, S., et al. 1999, ESO Messenger, 95, 8

Kratter, K. M., \& Matzner, C. D. 2006, MNRAS, 373, 1563

Krtička, J. 2014, A\&A, 564, A70

Kurtz, M. J., Eichhorn, G., Accomazzi, A., et al. 2000, A\&AS, 143, 41

Labadie-Bartz, J., Chojnowski, S. D., Whelan, D. G., et al. 2018, AJ, 155, 53

Langer, N., Baade, D., Bodensteiner, J., et al. 2020a, A\&A, 633, A40

Langer, N., Schürmann, C., Stoll, K., et al. 2020b, A\&A, in press, https:// doi.org/10.1051/0004-6361/201937375

Leone, F., \& Lanzafame, A. C. 1998, A\&A, 330, 306

Liu, J., Zhang, H., Howard, A. W., et al. 2019, Nature, 575, 618

Maintz, M. 2003, PhD Thesis Univ. of Heidelberg, Germany

Manchester, R. N., Hobbs, G. B., Teoh, A., \& Hobbs, M. 2005, AJ, 129, 1993

Moe, M., \& Di Stefano, R. 2017, ApJS, 230, 15

Nieva, M. F. 2013, A\&A, 550, A26

Nieva, M.-F., \& Przybilla, N. 2014, A\&A, 566, A7

Olejak, A., Belczynski, K., Bulik, T., \& Sobolewska, M. 2020, A\&A, in press, https://doi .org/10.1051/0004-6361/201936557

Perryman, M. A. C., \& ESA 1997, in The HIPPARCOS and TYCHO Catalogues. Astrometric and Photometric Star Catalogues Derived from the ESA HIPPARCOS Space Astrometry Mission, ESA Spec. Publ., 1200

Ricker, G. R., Winn, J. N., Vanderspek, R., et al. 2015, J. Astron. Telesc. Instrum. Syst., 1, 014003

Rivinius, T., Baade, D., \& Štefl, S. 2003, A\&A, 411, 229

Rivinius, T., Carciofi, A. C., \& Martayan, C. 2013, A\&ARv, 21, 69

Rivinius, T., Shultz, M. \& Wade, G. A. 2015, in New Windows on Massive Stars, eds. G. Meynet, C. Georgy, J. Groh, \& P. Stee, IAU Symp., 307, 228

Rodriguez, C. L., \& Antonini, F. 2018, ApJ, 863, 7

Shokry, A., Rivinius, T., Mehner, A., et al. 2018, A\&A, 609, A108

Slavin, J. D. 2017, in Structures in the Interstellar Medium Caused by Supernovae: The Local Bubble, eds. A. W. Alsabti, \& P. Murdin (Springer International Press), 2287

Slettebak, A. 1982, ApJS, 50, 55

Štefl, S., Hadrava, P., Baade, D., et al. 2004, in Stellar Rotation, eds. A. Maeder, \& P. Eenens, IAU Symp., 215, 166

Sukhbold, T., Ertl, T., Woosley, S. E., Brown, J. M., \& Janka, H. T. 2016, ApJ, 821,38

Thompson, T. A., Kochanek, C. S., Stanek, K. Z., et al. 2019, Science, 366, 637

Tokovinin, A., Everett, M. E., Horch, E. P., Torres, G., \& Latham, D. W. 2019, AJ, 158, 167

van Leeuwen, F. 2007, A\&A, 474, 653

Wang, L., Gies, D. R., \& Peters, G. J. 2018, ApJ, 853, 156

Wegner, W. 2002, Balt. Astron., 11, 1

Wegner, W. 2006, MNRAS, 371, 185

Wenger, M., Ochsenbein, F., Egret, D., et al. 2000, A\&AS, 143, 9 


\section{Appendix A: Radial velocities of the narrow-line} inner B3 III star and the mass function

Table A.1. Barycentric radial velocities of the inner B3 III star.

\begin{tabular}{|c|c|c|c|c|c|c|c|c|}
\hline \multirow{2}{*}{$\begin{array}{l}\text { BJD } \\
51373.172\end{array}$} & \multicolumn{2}{|c|}{$\begin{array}{c}\text { Radial velocity }\left[\mathrm{km} \mathrm{s}^{-1}\right] \\
\text { He I } \lambda 4026 \mathrm{Mg} \text { II } \lambda 4481\end{array}$} & \multirow{2}{*}{$\begin{array}{c}\text { BJD } \\
53162.357\end{array}$} & \multicolumn{2}{|c|}{$\begin{array}{l}\text { Radial velocity }\left[\mathrm{km} \mathrm{s}^{-1}\right] \\
\text { He I } \lambda 4026 \mathrm{Mg} \text { II } \lambda 4481\end{array}$} & \multirow{2}{*}{$\begin{array}{c}\text { BJD } \\
53244.049\end{array}$} & \multicolumn{2}{|c|}{$\begin{array}{c}\text { Radial velocity }\left[\mathrm{km} \mathrm{s}^{-1}\right] \\
\text { He I } \lambda 4026 \mathrm{Mg} \text { II } \lambda 4481\end{array}$} \\
\hline & -40.2 & -40.1 & & 42.8 & 40.1 & & 66.2 & 62.4 \\
\hline 51374.170 & -46.2 & -46.3 & 53183.043 & -44.8 & -45.5 & 53245.008 & 69.6 & 64.7 \\
\hline 51375.148 & -44.1 & -43.3 & 53185.046 & -50.8 & -51.5 & 53246.117 & 66.2 & 63.3 \\
\hline 51376.220 & -36.8 & -31.4 & 53187.161 & -53.5 & -53.0 & 53247.050 & 66.4 & 62.1 \\
\hline 51378.178 & -28.7 & -28.4 & 53188.182 & -52.9 & -52.2 & 53248.020 & 68.9 & 66.2 \\
\hline 51380.320 & -14.1 & -16.5 & 53190.199 & -48.8 & -50.0 & 53254.038 & 50.2 & 46.8 \\
\hline 51383.168 & 12.7 & 11.1 & 53194.186 & -26.8 & -28.4 & 53255.013 & 38.1 & 34.9 \\
\hline 51384.238 & 25.4 & 23.7 & 53195.058 & -13.4 & -16.5 & 53256.054 & 30.1 & 27.4 \\
\hline 51385.255 & 32.1 & 28.9 & 53196.046 & -1.3 & -3.8 & 53257.033 & 22.8 & 17.8 \\
\hline 51390.241 & 62.2 & 61.7 & 53197.165 & 10.0 & 6.6 & 53257.991 & 16.7 & 14.8 \\
\hline 51393.123 & 69.6 & 69.9 & 53199.065 & 27.4 & 25.2 & 53259.030 & 4.0 & 2.9 \\
\hline 51394.254 & 66.9 & 63.9 & 53202.140 & 52.9 & 49.0 & 53260.035 & -4.0 & -6.1 \\
\hline 53138.343 & -7.4 & -9.0 & 53204.008 & 68.2 & 63.9 & 53261.040 & -13.4 & -14.2 \\
\hline 53139.178 & -13.4 & -15.0 & 53205.192 & 72.9 & 66.9 & 53262.029 & -20.1 & -21.0 \\
\hline 53143.303 & -49.5 & -49.3 & 53206.996 & 75.6 & 70.6 & 53263.035 & -35.4 & -37.3 \\
\hline 53144.249 & -53.5 & -53.7 & 53226.093 & -51.5 & -51.5 & 53264.066 & -42.2 & -43.3 \\
\hline 53149.258 & -50.8 & -50.0 & 53229.987 & -46.2 & -44.0 & 53264.989 & -43.5 & -43.3 \\
\hline 53149.261 & -51.5 & -51.5 & 53231.066 & -39.5 & -40.3 & 53269.008 & -50.8 & -51.5 \\
\hline 53154.164 & -21.4 & -20.2 & 53238.992 & 20.1 & 18.5 & 53271.014 & -44.8 & -44.0 \\
\hline 53159.300 & 20.1 & 18.5 & 53240.099 & 30.1 & 28.2 & 53272.040 & -38.1 & -36.6 \\
\hline 53160.182 & 30.1 & 28.2 & 53243.012 & 59.5 & 54.5 & 53273.005 & -28.1 & -27.7 \\
\hline
\end{tabular}

Notes. BJD is Julian Date -2400000.5 .

Table A.1 lists the RVs measured by fitting single Gaussians to the narrow cores of the $\mathrm{He} \mathrm{I} \lambda 4026$ and $\mathrm{Mg}$ II $\lambda 4481$ lines. These cores move across the broad absorption profiles of the Be star, which are stationary at about the mean velocity of the B3 III star. Because this background flux increases toward both positive and negative velocities, the measured RV swing may be slightly reduced, which would tend to decrease the inferred minimum mass of the $\mathrm{BH}$.

The mass function (e.g., Batten 1973)

$f_{\mathrm{M}} \equiv \frac{P K_{1}^{3}}{2 \pi G}\left(1-e^{2}\right)^{3 / 2}=\frac{M_{\mathrm{BH}}^{3}}{\left(M_{\mathrm{B}}+M_{\mathrm{BH}}\right)^{2}} \sin ^{3} i$

can be solved iteratively for $M_{\mathrm{BH}}$ as

$\lim _{n \rightarrow \infty} M_{\mathrm{BH}, n+1}=\left(M_{\mathrm{B}}+M_{\mathrm{BH}, n}\right)^{2 / 3} f_{\mathrm{M}}^{1 / 3} \sin ^{-1} i$.

Considering the given values (see Table 2), this means that $M_{\mathrm{BH}}$ decreases with $M_{\mathrm{B}}$, but more slowly. For the following discussion, $\sin i=1$ is assumed to provide a limit. Evaluation of Eq. (A.2) for a grid of hypothetical values of $M_{\mathrm{B}}=$ $[3,5,7,9] M_{\odot}$ (which fully brackets the range for the B star, considering its spectrum, see below), returns minimum values of $M_{\mathrm{BH}}=[3.4,4.4,5.2,6.0] M_{\odot}$, respectively. In other words, for any plausible B star mass, the minimum mass ratio $M_{\mathrm{BH}} / M_{\mathrm{B}}$ is always close to unity, and for a B-star mass below about $3.8 M_{\odot}$, even rises to above one in favor of the unseen companion.

The analysis can be turned around by asking whether the minimum conventional $\mathrm{BH}$ mass of $3 M_{\odot}$ can be reconciled with a realistic mass of the B3 III star. For this, Eq. (A.1) can be solved for $M_{\mathrm{B}}$ directly as

$M_{\mathrm{B}}=\sqrt{\frac{M_{\mathrm{BH}}^{3} \sin ^{3} i}{f_{\mathrm{M}}}}-M_{\mathrm{BH}}$.
Substituting $3 M_{\odot}$ for $M_{\mathrm{BH}}$ leads to $M_{\mathrm{B}}=2.3 M_{\odot}$ (for $\sin i=1$, and even smaller for lower inclinations), a value that can be safely excluded because it is well below any conceivable mass of a B3 III star. As a consequence, if the companion does not produce nuclear energy in its core, and hence is luminous, it must be a BH and cannot be an NS or even a white dwarf. The discussion in Appendices C. 1 and C.2 therefore mainly focuses on the question of whether a luminous companion could have gone undetected.

\section{Appendix B: Space photometry of HR 6819}

HR 6819 (=QV Tel) was observed by the Transiting Exoplanet Survey Satellite (TESS, Ricker et al. 2015) in Sector 13 for $\sim 28.4$ days $^{3}$. This is too short for a formal period search in the range of the $40 \mathrm{~d}$ spectroscopic period (Table 2). However, phase-folding the data with this period did not indicate a coherent variability on that timescale.

On timescales starting at about half a day, the system is variable with a peak-to-peak amplitude of nearly 70 mmag (Fig. B.1, top panel). At B3 III, the inner B star may be a slowly pulsating B star (SPB star; an example is 18 Peg (B3 III): Irrgang et al. 2016) and the outer Be star may also be pulsating (Rivinius et al. 2003). Typical pulsation amplitudes rarely exceed 10 mmag. From Be stars, much larger amplitudes are known and can often be traced to matter ejected into the inner regions of the equatorial disk when viewed at small to intermediate angles (intermediate to large inclination angles of the stellar rotation axis; Baade et al. 2016, 2018). The appearance of the $\mathrm{H} \alpha$ emission-line profile

3 Data available from the Mikulski Archive for Space Telescopes (MAST): https ://dx.doi.org/10.17909/t9-tejq-s571 

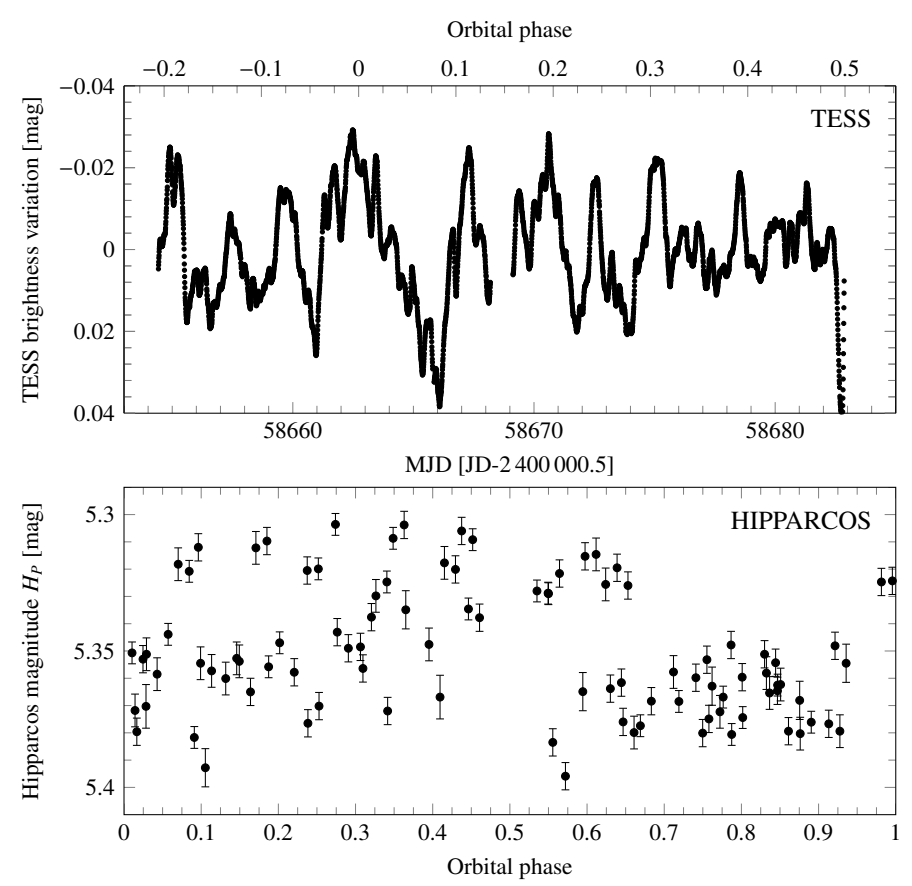

Fig. B.1. Photometric data from TESS (upper panel) and HIPPARCos (lower panel, folded with the orbital period of $40.333 \mathrm{~d}$, see Table 2) The TESS photometry spans less than one orbital cycle, so that both Julian date and phase are indicated on the axes.
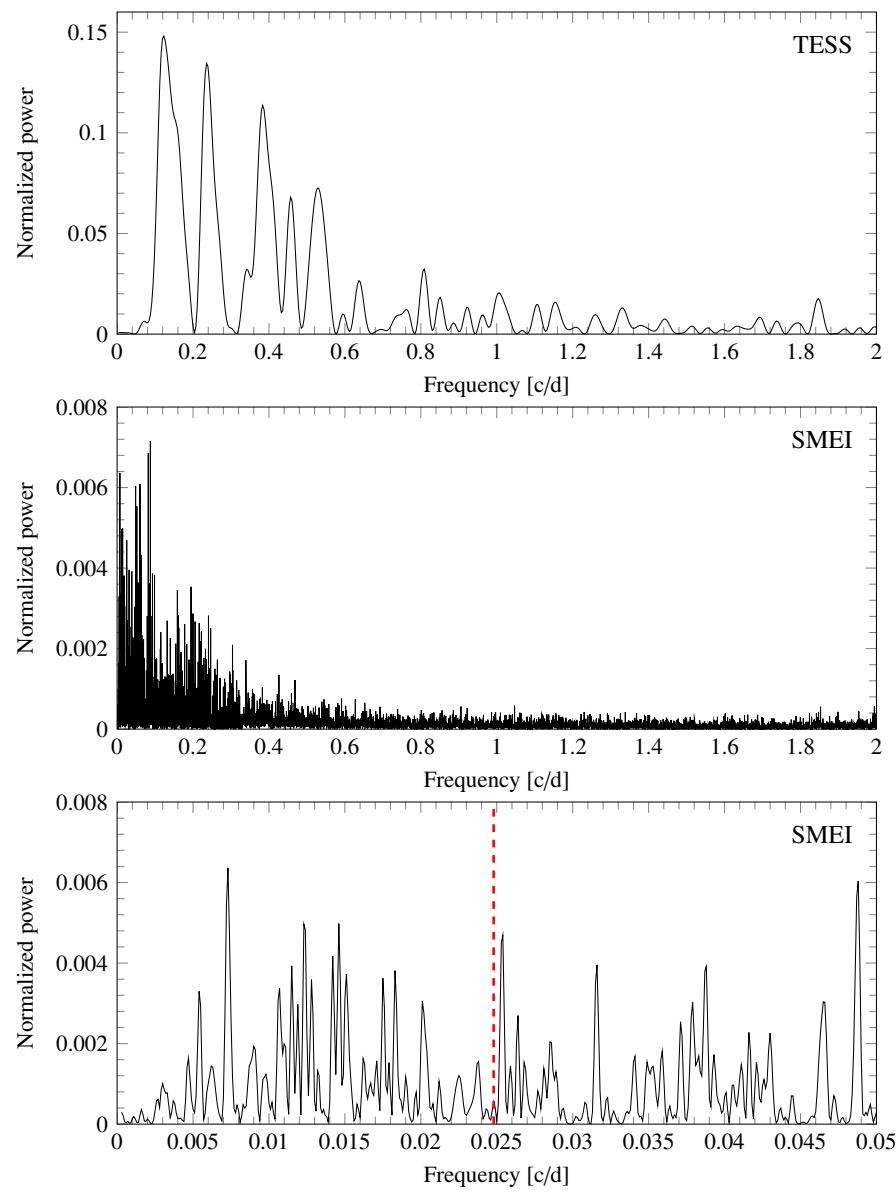

Fig. B.2. Periodograms of TESS and SMEI photometry (as labeled). Bottom panel: SMEI periodogram around the orbital frequency, marked by a vertical dashed line.
(Fig. 2) clearly indicates an orientation of the disk not far from face-on (Hummel 1994; Rivinius et al. 2013). Because the Be star probably only accounts for little over half of the total optical flux (Appendix C), it would be fairly active, which is not unusual for early-type Be stars (e.g., Labadie-Bartz et al. 2018; Bernhard et al. 2018). This large-amplitude circumstellar variability probably dominates any pulsational signatures of the two luminous stars (see Fig. B.2, upper panel).

HIPPARCOs epoch photometry (Perryman \& ESA 1997) does not indicate any sign of the orbital period either and is not suitable to study shorter timescales, even though the variability as such is clearly visible (Fig. B.1, bottom panel). Data obtained with the Solar Mass Ejection Imager (SMEI, Howard et al. 2013) paint a similar picture: Although the amplitude observed by TESS and HIPPARCOS is within the reach of SMEI (Hick et al. 2007), no signal clearly linked to the orbit emerges from the SMEI observations either (see Fig. B.2, lower two panels).

\section{Appendix C: Stellar parameters adopted for the inner B star and the implied distance to the system}

\section{C.1. Minimum masses in the inner system}

The star that is part of the inner binary has been classified as B3 III on account of its sharp lines (see Slettebak 1982). The outer Be star stands out by its emission lines, but the shallowness of the broad photospheric absorption lines prevents a direct classification other than being similar to that of the inner B star. While in an unrecognized composite spectrum, the accuracy of spectral classifications can be compromised, in HR 6819 there are good arguments that both temperature and luminosity of the inner B star are close to B3 III. The spectral typing is relevant for this work because the minimum mass of the unseen object depends, through the mass function, on the minimum mass of its narrow-line companion in the inner binary. When we consider the isolines in the Hertzsprung-Russell diagram, determining the minimum mass of a star requires determining at least a minimum temperature or a minimum luminosity, and preferably both.

For a minimum temperature, ratios of lines from the same element but different ionization stages can be good indicators. Strong Fe II and Fe III lines of the narrow component lie in the range between 510 and $520 \mathrm{~nm}$. The Fe II $\lambda 5169$ is stronger than Fe III $\lambda \lambda 5127,5156$ by about a factor of two. For this line ratio, synthetic ATLAS9/SYNSPEC spectra (Shokry et al. 2018) show a monotonic growth of the temperature from $16 \mathrm{kK}$ for $\log g \sim 2.5$ to $18 \mathrm{kK}$ for $\log g \sim 3.5$ (see Fig. C.1). This places the narrow-line inner B star indeed in the B3 range (e.g., Hohle et al. 2010, assume $17100 \mathrm{~K}$ as a typical $T_{\text {eff }}$ for a B3 III star in their calibration).

Similar arguments can be made for the luminosity class of the narrow-line component. As a first step, it is important to note that He I lines are insensitive to temperature in the spectral range B2-B3. By contrast, they are sensitive to gravity, and hence luminosity, through the increasing pressure broadening as $\log g$ increases (Leone \& Lanzafame 1998).

The He I lines of the inner B3 III star are less broadened than those of the Be star. This is revealed by the difference in RV variability between the two stars: The outer limits of the blends of the pressure-broadened He I lines do not participate in the RV variation of the metal lines (see He I $\lambda \lambda 4026,4388$ in Fig. C.2). Because the Be star does not exhibit any noticeable RV variations (as is evident from the quasi-stationary line emission), the 


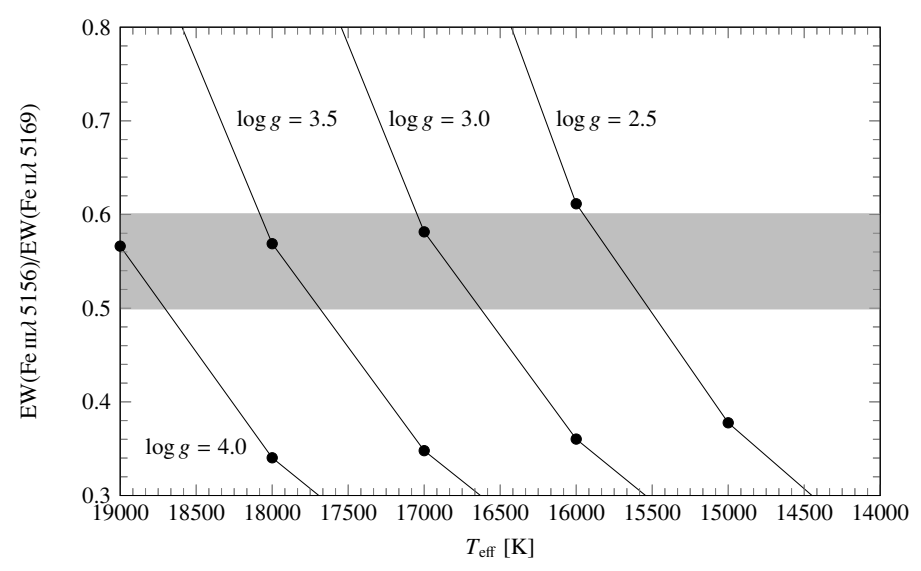

Fig. C.1. Fe III $\lambda 5156$ to Fe II $\lambda 5169$ equivalent-width ratio vs. effective temperature $\left(T_{\text {eff }}\right)$. Measurements in theoretical spectra (the nonrotating input grid used by Shokry et al. 2018) are shown as lines for various values of $\log g$ (as labeled). The range observed in the inner (narrow line) B star of HR 6819 is shaded in gray. This diagnostic diagram suggests that $T_{\text {eff }}$ lies between 16 and $18 \mathrm{kK}$. A more precise value is difficult to give owing to blending with the Fe II $\lambda 5169$ emission line of the Be star (see Fig. 2).

also stationary outer wings of the $\mathrm{He}$ I lines must be due to the $\mathrm{Be}$ star. Because the stationary wings are wider, the pressure broadening of the Be star is higher. By implication, the lower broadening of the inner B star indicates a lower gravity, that is, higher luminosity. Accordingly, the inner B star is more evolved than the Be star.

An upper bound on the luminosity can be deduced from the presence or absence of emission lines that form deep in the photosphere (Rivinius et al. 2015). They become stronger as the photospheric density decreases, that is, the scale height and luminosity increase. In HR 6819, such emission lines are present at $\lambda \lambda \sim 6240 \AA$ (a group of four lines), $7513 \AA$, and $7849 \AA$ (see Fig. C.2 for an example). Candidate identifications of the two latter features are Fe II $\lambda 7513.1767$ and the Si II $\lambda \lambda 7848.80,7849.72$ doublet. Some of these lines are also present in BW Vul and $\xi^{1} \mathrm{CMa}$, which have spectral types B2 III and B0.7 IV, respectively (Rivinius et al. 2015). No other such emission lines were found in HR 6819. Because these emission lines become stronger and more abundant at higher luminosity, a luminosity class of IV-III is suggested for the inner B star, and that of the Be star is V.

In conclusion, the spectrum of the inner narrow-line star agrees with a $16-18 \mathrm{kK}$ star toward the end of the main sequence. In the evolutionary tracks studied by Ekström et al. (2012), for instance, this means a star of at least $5 M_{\odot}$, and most likely of higher mass. Supportive evidence is available from Hohle et al. (2010), who listed masses for 56 B3 III stars; none of them has a median value lower than $5 M_{\odot}$. Therefore, $5 M_{\odot}$ is a robust minimum mass for the inner B3 III star that is suitable to establish a similarly robust minimum mass of the unseen companion.

\section{C.2. Limits on the companion detectability}

The previous section considered the question of the minimum mass of the inner system and concluded that a second luminous star therein with the found minimum mass could not remain undetected. As is seen from the structure of the mass function (Appendix A), however, not only the minimum mass is important, but the mass difference between the B star and its com- panion matters as well. This difference increases with increasing B star mass, and the associated larger difference in luminosity would make it easier for a luminous companion to hide. The median mass values of Hohle et al. (2010) used above for the determination of the upper mass limit for the B3 III star include two outliers at about $10 M_{\odot}$ while all others are below $8 M_{\odot}$. With an analogous reckoning to that used in Sect. 3.1 above, this suggests a minimum mass of the companion of the unseen companion of $5.6 M_{\odot}$.

Hohle et al. (2010, for the masses) and Wegner (2006, for the absolute magnitudes, see also Sect. 3.1 for an explanation) implied that for any combination of main-sequence masses between 5 and $4.2 M_{\odot}$ (minimum masses of the two inner system companions as derived in Sect. 3.1) and 8 and 5.6 $M_{\odot}$ (maximum masses, as derived above in this section), respectively, the magnitude difference $\Delta M_{V}$ does not exceed $1.7 \mathrm{mag}$. It is larger for the lower set of masses. As we discuss below, it is not plausible that a companion with such a small magnitude difference could be missed in the available data, even for a critically rotating companion.

\section{C.3. Parameters relative to similar $B$ stars}

The previous subsection aimed at deriving the minimum mass of the inner B3 III star and, thereby, that of the unseen component. However, more realistic properties that are not based on skewed choices toward a minimum mass are needed for the use of the inner B star as the pivotal element for the analysis of all other properties, especially those of the Be star. This is achieved best by comparison to calibrations from larger samples, or analyses of individual similar stars. The following will pursue both avenues. Any systematic errors for the inner B3 III star will propagate into similar systematic errors for the Be star, but will leave all differential conclusions intact. Independent absolute analyses of the two stars would achieve this less easily.

The calibration of B3 III stars performed by Hohle et al. (2010, based on multicolor photometry, HIPPARCOS parallaxes, and different evolutionary models for 68 B3 III stars) gives a mean mass of $6.31 \pm 0.7 M_{\odot}$ with an assumed $T_{\text {eff }}=17100 \mathrm{~K}$ for B3 III stars. A broader range of physical parameters was derived for the B3 III star 18 Peg by Nieva \& Przybilla (2014, object 15 in their list). They found $5.8 \pm 0.4 M_{\odot}$ and also determined the temperature $\left(T_{\text {eff }}=15.8 \pm 0.2 \mathrm{kK}\right)$, gravity $(\log g=3.75 \pm 0.05)$, radius $\left(5.5 \pm 0.5 R_{\odot}\right)$, and evolutionary age $\left(10^{7.8 \pm 0.1} \mathrm{Myr}\right)$. The SB1 nature of $18 \mathrm{Peg}$ (see below) was not known at the time. However, from the absence of any spectroscopic signature of a possible secondary, Irrgang et al. (2016) placed an upper limit of only $7 \%$ on the flux contribution by a secondary at visual wavelengths.

In their dedicated study of 18 Peg, Irrgang et al. (2016) derived the parameter values photometrically, which in particular concerning gravity, is less reliable than modern spectroscopic methods. Their rather low value of $\log g(3.41 \pm 0.15)$ is reflected in a surprisingly high radius $\left(8.4 \pm 1.6 R_{\odot}\right)$ and luminosity $\left(4000 \pm 1400 L_{\odot}\right)$. The calculated mass is $6.9 \pm 0.7 M_{\odot}$. It is noteworthy that according to the note to Table 1 of Irrgang et al. (2016), these values were obtained using the evolutionary tracks of Ekström et al. (2012). However, as we show in the next section in Fig. D.1, they are only compatible with a star that is in the rapidly progressing expansion phase toward the giant branch. The parameters derived in the same study by asteroseismology $\left(\log g=3.22 \pm 0.01,10.9 \pm 0.15 R_{\odot}\right)$ are even farther off, and Irrgang et al. (2016) pointed out that the stated uncertainties only reflect the statistical part, not any potential systematic 

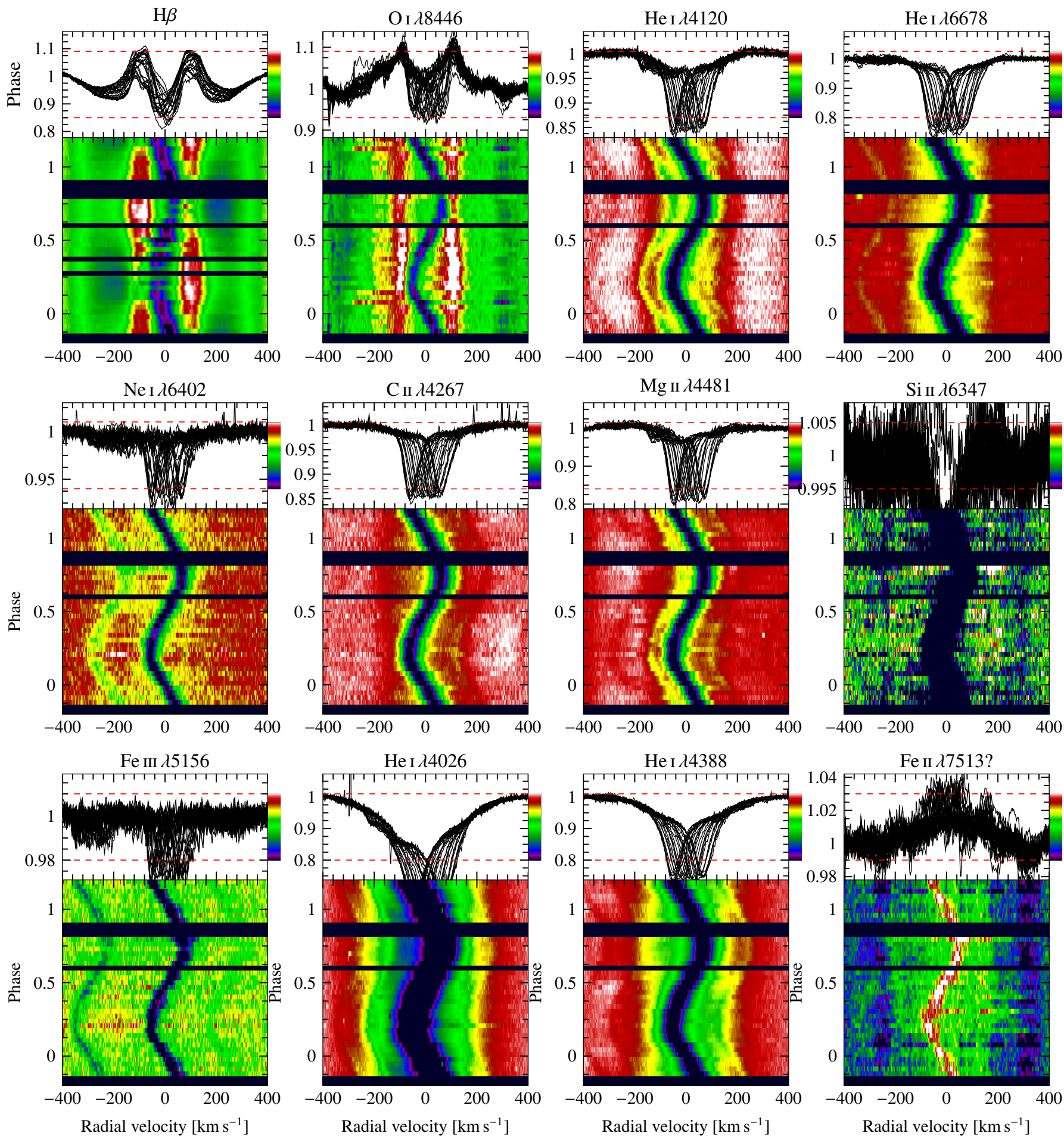

Fig. C.2. Additional (see Fig. 2) dynamical spectra of selected circumstellar and photospheric lines (as labeled) of the HR 6819 system, phased with the orbital period (see Table 2) of the inner binary. The individual line profiles are overplotted at the top of each panel, where dashed horizontal lines identify the ranges of the color-coding in the dynamical spectra. $\mathrm{H} \beta$ was constructed with dataset $\mathrm{B}$ alone (see Table 1 ) to minimize the effect of the long-term disk variability of the Be star on the appearance of the emission.

offsets. Adoption of the evolutionary tracks by Brott et al. (2011) might lead to a more plausible star. However, considering that 18 Peg is only used as a proxy for a typical B3 III star and the parameter values from Nieva \& Przybilla (2014) agree reasonably well with the calibration by Hohle et al. (2010), these values appear adequate for the purpose of characterizing the B3 III star in HR 6819. Therefore, while $5 M_{\odot}$ is the absolute minimum mass of the inner B star (Appendix C.1), the range of 6-7 $M_{\odot}$ is more suitable for all other purposes.

\section{C.4. Distance to the system}

With these parameters, distance can be addressed. Neither Gaia nor HIPPARCos parallaxes take binarity into account, and 


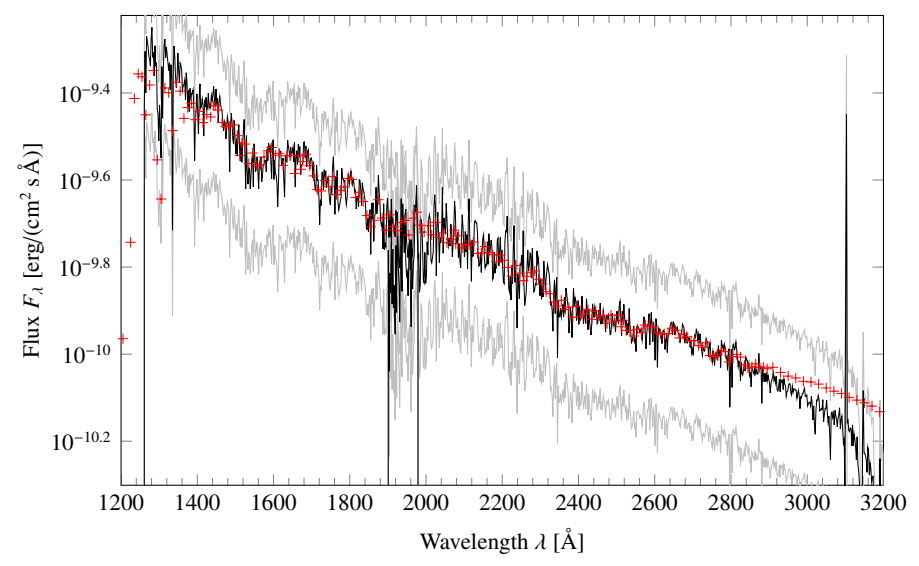

Fig. C.3. Binned and dereddened IUE spectra (black) of HR 6819. They are scaled to $55 \%$ to represent the flux contribution from the inner B3 III component to the total flux; spectra plotted in gray indicate $35-75 \%$ ranges (Appendix C.4.) The theoretical SED for $T_{\text {eff }}=16 \mathrm{kK}$ and $\log g=3.5$ (Castelli \& Kurucz 2003) is overplotted in red, multiplied by $6.2 \times 10^{-18}$ to account for distance and stellar radius.

therefore do not reach their nominal accuracy (Sect. 2.3). An alternative distance estimate can be made with UV fluxes measured with the International Ultraviolet Explorer (IUE) satellite (Boggess et al. 1978) ${ }^{4}$. IUE obtained two high-resolution spectra, one in the long- and one in the short-wavelength range. Both observations used the large aperture so that they are suitable to determine absolute fluxes. The spectra were resampled in a flux-conserving way with a $2 \AA$ bin to reduce the noise. For the dereddening of the measured fluxes, the reddening $E(B-V)=0.135$ mag listed by Wegner (2002) for HR 6819 and the extinction parameterization by Cardelli et al. (1989) were applied together with the standard value of the Galactic totalto-selective extinction ratio, $R_{V}=3.1$.

Model fluxes corresponding to the parameters estimated above are available from Castelli \& Kurucz (2003, it should be noted that these were adapted to $F_{\lambda}$ surface flux units $)^{5}$, although only for $T_{\text {eff }}=16 \mathrm{kK}$ and $\log g=3.5$ and 4.0. For the comparison here, $\log g=3.5$ was chosen, but the difference to 4.0 is marginal in view of the other uncertainties discussed below.

Because HR 6819 is a multiple star, the flux contributions by each component have to be taken into account. Preliminary results from disentangling the spectra indicate that the inner B3 III and the outer Be star are about equally bright in the visual regime and that the Be star has a slightly higher temperature and a somewhat higher $\log g$ (Hadrava et al., in prep.). The similarity in flux is corroborated by the equivalent widths (EWs) of He I lines of the two stars when lines with only a small contribution from pressure broadening are chosen. Across the given temperature and luminosity domain, they are fairly constant, so that the EW ratio is a good proxy of the luminosity ratio (Leone \& Lanzafame 1998). For instance, the total EW of He I $\lambda 4713$ is about $0.3 \AA$, to which the narrow component alone contributes 50 to $60 \%$. That is, the two stars are about equally bright, and a contribution to the total IUE flux by the B3 III component of $55 \pm 20 \%$ seems a reasonable estimate.

\footnotetext{
4 Data available from the Mikulski Archive for Space Telescopes: https://dx.doi.org/10.17909/t9-n3zj-zv42

5 Downloadable from: http://www.stsci.edu/hst/ instrumentation/reference-data-for-calibration-andtools/astronomical-catalogs/castelli-and-kurucz-atlas
}

Figure C.3 depicts the observed absolute UV flux distribution of the HR 6819 system as characterized above and scaled to $55 \%$ for comparison to the theoretical Castelli \& Kurucz model of the inner B3 III star alone. The slope indicates a slightly hotter overall flux distribution (i.e., the sum of the modeled inner B3 III and the outer Be star), which agrees with the preliminary disentangling solution of a slightly hotter outer Be star. The match of observed IUE flux and theoretical spectral energy distribution (SED) can also be appreciated in Fig. C.3.

For the flux definition of the Castelli \& Kurucz (2003) grid as offered by the STScI (see above), distances $D$ are simply obtained as $D=R \sqrt{f_{\mathrm{s}}}$, where $R$ is the stellar radius and $f_{\mathrm{s}}$ is the distance-dependent dimensionless flux-scaling factor, which for the B3 III star HR 6819 is $f_{\mathrm{s}}=6.2 \pm 1 \times 10^{18}$ (see Fig. C.3). Considering that the uncertainties of the result are currently fully dominated by other factors such as the flux ratio of the components (as seen by the range between the gray SEDs indicated in Fig. C.3), a simple visual approximation was sufficiently precise to derive $f_{\mathrm{s}}$. Adopting $5.5 \pm 0.5 R_{\odot}$ as discussed above results in a distance to the system of $310 \pm 60 \mathrm{pc}$. The uncertainty is dominated by the loosely constrained flux distribution between the two luminous components in the system, with the second largest contribution coming from the stellar radius. Overall, the larger the relative flux contribution by the B3 III component, the more nearby the system, while the distance would increase with the B3 III radius.

Alternative plausible values for temperature, radius, reddening, and selective absorption as well as fitting the observed SED with two theoretical SEDs with flux ratios in the above range (35-75\%) alter the distance and its uncertainty only marginally: While the distance changes by less than a dozen parsec, the uncertainty range is always several dozens of parsec. This uncertainty is again dominated by the flux ratio between the two stars. Only with a better constrained flux ratio can a more precise distance value be derived from the SED.

\section{Appendix D: Properties of the outer Be star and the age of the system}

As illustrated by Fig. 2 and preliminarily quantified in Appendix C.4 through the EW of He I 4713, the outer Be star is of similar spectral type and similar visual magnitude as the inner B3 III star. This is also supported by the disentangling of spectra (Hadrava et al., in prep.). However, this similarity does not imply that the Be star itself shares the other parameter values of the inner star as well because the observed flux is the sum of the stellar and a circumstellar component. There is a well-known magnitude excess in Be stars that is caused by scattering of stellar light in the circumstellar disk, of up to $\Delta V \sim-0.5 \mathrm{mag}$ (Haubois et al. 2012). Because the Be star in HR 6819 has a strong $\mathrm{H} \alpha$ emission and low inclination, as follows from the shape of the emission (see Hummel \& Vrancken 2000; Rivinius et al. 2013), this upper limit is adopted with a conservative range of -0.3 to $-0.7 \mathrm{mag}$. Rapid rotation is another mechanism that may alter the apparent parameters of a star in an aspect-angle-dependent way (Rivinius et al. 2013). However, the effect is too small in comparison to other uncertainties to be considered here.

Figure D.1 identifies the potential location of the Be star in HR 6819 (i.e., after correction for the circumstellar flux excess) relative to a B3 III star in a theoretical HertzsprungRussell diagram (HRD), along with evolutionary tracks and isochrones. The underlying values correspond to those derived for 18 Peg by Nieva (2013) and by Irrgang et al. (2016) and to the 


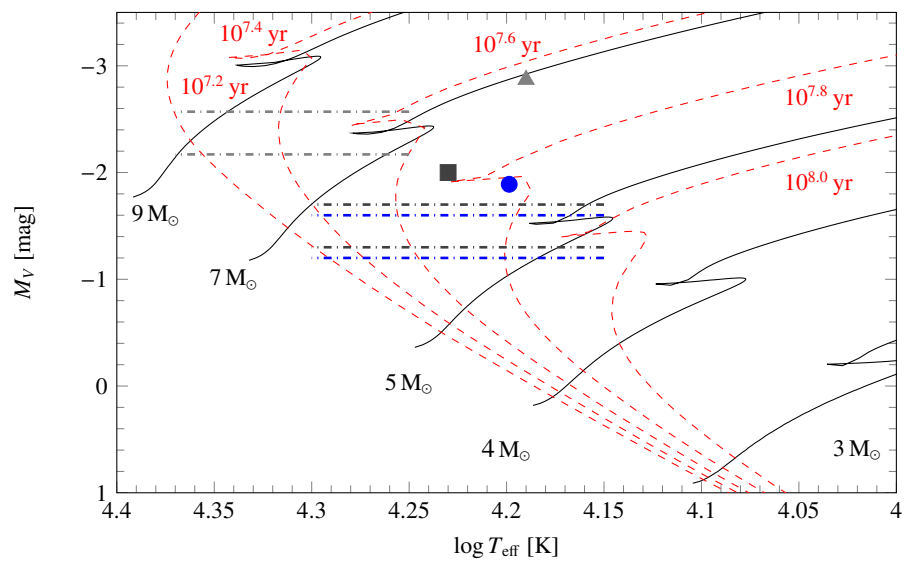

Fig. D.1. Evolutionary tracks (solid) and isochrones (dashed) from Ekström et al. (2012). Three potential locations of B3 III stars are marked by filled shapes: 18 Peg as modeled by Nieva \& Przybilla (2014, blue filled circle) as well as by Irrgang et al. (2016, gray triangle), and the generic calibration by Hohle et al. (2010, dark gray square), all discussed in Appendix C. The horizontal dash-dotted lines give the respective implied ranges of the intrinsic brightness of the Be star in HR 6819. The blue and dark gray lines assume that the apparent magnitude of the Be star is the same as that of a B3 III star as determined by Nieva \& Przybilla (2014) and Hohle et al. (2010), respectively. Each pair of lines with the same color indicates a disk excess $\Delta V$ of -0.3 and -0.7 mag, respectively.

general calibration by Hohle et al. (2010), see also Appendix C. The choice of 18 Peg does not pretend to present the true values for HR 6819, but merely serves as an anchor for the discussion of the values of temperature, luminosity, and age of the Be star in HR 6819 relative to the inner B3 III star. The luminosities, $L$, were converted into absolute visual magnitudes, $M_{V}$, using Eq. (10) of Nieva (2013) for the bolometric correction, so that for a single star with temperature $T_{\text {eff }}$

$M_{V}=-2.5 \log L+4.74-\left(21-5.34 \log T_{\text {eff }}\right)$.

No correction is included for binarity, and the calculated $M_{V}$ is independent of reddening, which has to be accounted for when the apparent magnitude is derived from the absolute magnitude. In the following discussion, the properties of the Be star refer to the Be star alone (without contributions from the circumstellar disk to the brightness).

The evolutionary age of B3 III stars is about $65 \pm 15 \mathrm{Myr}$ (see the result for 18 Peg by Nieva \& Przybilla 2014, which is based on computations by Ekström et al. 2012). When similar values as by Nieva \& Przybilla (2014) are assumed and both stars in HR 6819 evolved unaffected by binary interaction, the isochrones in Fig. D.1 (dark gray symbols) suggest a common age of about 70 Myr. However, when instead parameter values such as those by Irrgang et al. (2016) are assumed, the individual evolutionary ages of the two stars are less easily reconciled under the assumption of single star evolution for both stars, namely with an age of about $40 \mathrm{Myr}$, but the Be star would have to be at the very end of its core hydrogen-burning phase, and the B star well beyond it (see Fig. D.1, light gray symbols).

The so-called Be phenomenon (Rivinius et al. 2013) is limited to the main sequence (taken as the core hydrogen-burning phase, which for B stars, includes luminosity classes V to III). After core-hydrogen exhaustion, expansion of the envelope causes the surface rotation to slow down far below the nearcritical values that are needed for a Be star to form and sustain a Keplerian disk. With parameter values similar to those obtained by Nieva \& Przybilla (2014), a B3 III star is about to reach the end of its main sequence life. It follows that a star of equal age cannot have both similar $M_{V}$ and significantly lower $T_{\text {eff }}$ or later spectral type (Fig. D.1) because it would fall well above the main sequence. Preliminary disentangling of the spectra, as well as the relative line strengths over the full instrumental wavelength range, indeed indicate that the Be star may be slightly hotter than the B3 III star and also have a slightly higher surface gravity. Figure D.1 therefore shows that the Be star must be on the same or on an earlier isochrone, and the B3 III must be at least as old as the Be star. Only if there had been significant binary interaction in the inner system could the B3 III appear younger than the Be star because it would have acquired additional fuel and lifetime through Roche-lobe overflow from the $\mathrm{BH}$ progenitor.

The current orbital separation of the inner binary makes it likely that the two stars have been interacting. If the evolutionary age of the B3 III star nevertheless remained unaltered or even increased relative to the Be star, more remote explanations need to be considered. For instance, it appears conceivable that hydrogen-depleted material from the $\mathrm{BH}$ progenitor was transferred to the core of the inner B star, thereby reducing the operating time of the inner B star. Alternatively, if the Be star is itself a binary, it might have been rejuvenated as well and by more than the B3 III star. This is not completely implausible: If Be stars owe their rapid rotation to spin-up by mass overflow from a now underluminous companion (e.g., Langer et al. 2020a, and references therein) a resulting rejuvenation of the outer Be star is one possible explanation. Some blue stragglers in open clusters have been reported to show $\mathrm{H} \alpha$ in emission and may be classical Be stars (Aidelman et al. 2018; Bodensteiner et al. 2020).

When we disregard the possible difference between the age estimates, the outer Be star considered alone might be as young as $\sim 10^{7.2}=15 \mathrm{Myr}$ if the preliminary "slightly hotter and slightly higher gravity" result from the disentangling is taken at face value. A conclusive statement on this problem has to await the results of the full disentangling of the spectra of the two stars (Hadrava et al., in prep.). Under the current premises, the age bracket of 15 to $75 \mathrm{Myr}$ for the HR 6819 system is the most plausible scenario, with a preference for the higher value. 